\title{
Defining an international cut-off of two-legged countermovement jump power for sarcopenia and dysmobility syndrome
}

\author{
N. Hong ${ }^{1} \cdot$ E. Siglinsky ${ }^{2,3} \cdot$ D. Krueger ${ }^{2} \cdot$ R. White ${ }^{2} \cdot$ C. O. Kim ${ }^{4} \cdot$ H. C. Kim ${ }^{5} \cdot$ Y. Yeom ${ }^{6} \cdot$ N. Binkley ${ }^{2} \cdot$ Y. Rhee ${ }^{1} \cdot$ \\ B. Buehring ${ }^{2,7}$
}

Received: 10 March 2020 / Accepted: 10 July 2020 / Published online: 7 September 2020

(C) The Author(s) 2020

\begin{abstract}
Summary We aimed to establish jump power cut-offs for the composite outcome of either sarcopenia (EWGSOP2) or dysmobility syndrome using Asian and Caucasian cohorts. Estimated cut-offs were sex specific (women: < $19.0 \mathrm{~W} / \mathrm{kg}$; men: $<23.8 \mathrm{~W} / \mathrm{kg}$ ) but not ethnicity specific. Jump power has potential to be used in definitions of poor musculoskeletal health.

Purpose Weight-corrected jump power measured during a countermovement jump may be a useful tool to identify individuals with poor musculoskeletal health, but no cut-off values exist. We aimed to establish jump power cut-offs for detecting individuals with either sarcopenia or dysmobility syndrome.

Methods Age- and sex-matched community-dwelling older adults from two cohorts (University of Wisconsin-Madison [UW], Korean Urban Rural Elderly cohort [KURE], 1:2) were analyzed. Jump power cut-offs for the composite outcome of either sarcopenia defined by EWGSOP2 or dysmobility syndrome were determined.

Results The UW $(n=95)$ and KURE $(n=190)$ cohorts were similar in age (mean 75 years) and sex distribution $(68 \%$ women). Jump power was similar between KURE and UW women (19.7 vs. 18.6 W/kg, $p=0.096)$ and slightly higher in KURE than UW in men (26.9 vs. $24.8 \mathrm{~W} / \mathrm{kg}, p=0.050)$. In UW and KURE, the prevalence of sarcopenia (7.4\% in both), dysmobility syndrome (31.6\% and $27.9 \%$ ), or composite of either sarcopenia or dysmobility syndrome (32.6\% and $28.4 \%$ ) were comparable. Low jump power cut-offs for the composite outcome differed by sex but not by ethnicity $(<19.0 \mathrm{~W} / \mathrm{kg}$ in women; $<23.8 \mathrm{~W} / \mathrm{kg}$ in men). Low jump power was associated with elevated odds of sarcopenia (adjusted odds ratio [aOR] 4.07), dysmobility syndrome (aOR 4.32 ), or the composite of sarcopenia or dysmobility syndrome (aOR 4.67, $p<0.01$ for all) independent of age, sex, height, and ethnicity.

Conclusion Sex-specific jump power cut-offs were found to detect the presence of either sarcopenia or dysmobility syndrome in older adults independent of Asian or Caucasian ethnicity.
\end{abstract}

Keywords Countermovement jump $\cdot$ Dysmobility syndrome $\cdot$ Jump power $\cdot$ Sarcopenia

Electronic supplementary material The online version of this article (https://doi.org/10.1007/s00198-020-05591-x) contains supplementary material, which is available to authorized users.

\section{B. Buehring}

bjoern.buehring@elisabethgruppe.de

Y. Rhee

yumie@yuhs.ac

1 Department of Internal Medicine, Severance Hospital, Endocrine Research Institute, Yonsei University College of Medicine, Seoul, Korea

2 Osteoporosis Clinical Research Program, Department of Medicine, University of Wisconsin School of Medicine and Public Health, 2870 University Avenue, Suite 100, Madison, WI 53705, USA
3 UT Southwestern Medical Center, University of Texas Southwestern, Dallas, TX, USA

4 Division of Geriatrics, Department of Internal Medicine, Severance Hospital, Yonsei University College of Medicine, Seoul, Korea

5 Department of Preventive Medicine, Yonsei University College of Medicine, Seoul, Korea

6 Department of Sociology, Yonsei University College of Social Sciences, Seoul, Korea

Rheumazentrum Ruhrgebiet, Ruhr-Universität Bochum, Herne, Germany 


\section{Introduction}

Sarcopenia, the age-related loss of muscle mass and function, is recognized as an important geriatric syndrome leading to negative health outcomes such as falls, fractures, hospitalizations with increased medical costs, and mortality [1-5]. Until recently, low muscle mass was required to diagnose sarcopenia as a core component, despite evidence suggesting muscle function as an independent and more sensitive indicator for aging and health outcomes [3, 6-8]. Taking this into consideration, the European Working Group on Sarcopenia in Older People (EWGSOP) proposed a new algorithm with the emphasis on screening for sarcopenia in individuals with low muscle function rather than muscle mass [3]. As a more comprehensive approach, dysmobility syndrome was previously proposed to identify older individuals with high risk of falls, fractures, and decreased mobility $[4,9,10]$. Similar to metabolic syndrome, dysmobility syndrome is diagnosed when several components are present together including previous falls, low muscle function, poor physical performance, low appendicular lean mass, high fat mass, and low bone mass $[10,11]$. In Caucasian and Asian middle-aged to elderly cohorts, dysmobility syndrome is associated with decreased physical activity and increased risk for falls, fractures, and mortality [12-16].

Establishing a diagnostic threshold for muscle function testing with good discriminatory ability is important for the identification of individuals with sarcopenia or dysmobility syndrome. Currently, the most commonly used muscle function tests in sarcopenia definitions are usual gait speed and grip strength $[3,4,6,17,18]$. Both usual gait speed and grip strength have proven their usefulness in large epidemiologic studies but have limitations in a given individual including larger variability, not testing maximal effort (and therefore having a ceiling effect), being impacted by comorbidities and testing maximal force rather than power $[4,6,17,19]$. Muscle function tests that do not have these limitations may have the potential to improve diagnostic performance for identifying individuals with sarcopenia or dysmobility syndrome and also monitoring disease progression or response to treatment over time. Jump power, measured during a two-legged countermovement jump, has been utilized to assess muscle function in various populations including older adults with impaired function [20-28]. The countermovement jump combines maximal effort during an explosive movement (i.e., high power output) with the requirement for good coordination of various muscle and joint groups, and organ systems required for balance $[4,19,20]$. It combines features of classical muscle function tests like grip strength (maximal effort) with classical physical function tests like usual gait speed (requirement for a high degree of coordination of different organ systems), with good safety and reproducibility $[4,20,21,28,29]$. Low jump power is associated with sarcopenia or dysmobility syndrome in older Caucasian or
Asian adults [24, 30, 31]. However, to our knowledge, international cut-offs for weight-corrected jump power to identify individuals with either sarcopenia or dysmobility syndrome across different ethnic groups have not been investigated. The aim of this study was to explore the cut-offs for weight-corrected jump power that could be used internationally to identify individuals with sarcopenia or dysmobility syndrome using a combined cohort of Caucasian and Asian older adults.

\section{Methods}

\section{Cohorts}

For this analysis, we combined cohorts of older communitydwelling individuals with two different ethnic backgrounds (Asian and Caucasian) in whom data on muscle mass and function were available.

\section{Korean urban rural elderly cohort (South Korea)}

The KURE study is a prospective, longitudinal cohort study investigating health and aging of community-based older adults in Korea. A total of 3517 individuals residing in three urban districts and one rural area were recruited from 2012 to 2015 via random visits, local government health facilities, or promotional posters. Further details of the KURE protocol can be found elsewhere $[32,33]$. This study was approved by the Severance Hospital Ethics Committee (IRB No. 4-20120172). Because jumping mechanography and bioimpedance analysis (BIA) testing were added to the standard study protocol of KURE in 2014, only participants recruited in 2014 and 2015 (1493 subjects) were included in this study. All participants underwent anthropometric analysis, interviewerassisted questionnaires, laboratory assessments, and dualenergy x-ray absorptiometry (DXA, QDR 4500A; Hologic, USA) for bone mineral density measurements according to standardized protocols. Appendicular lean mass was estimated through BIA (InBody720; Biospace Co., Ltd., Seoul, Korea). All participants were asked to perform muscle function tests including timed get-up-and-go, grip strength, and two-legged jumping on a computerized ground force plate. Because 4-m gait speed testing was added to the standard examination protocol in the middle of the follow-up phase (2018), estimated gait speed was calculated from timed getup-and-go results using conversion equation derived from an internal dataset (KURE dataset 2018, $n=605$; gait speed $[\mathrm{m} / \mathrm{s}]=4 /[$ timed get-up-and-go performance (second) $\times$ $0.360+0.146]$; Supplementary Fig. 1). Those who had active inflammatory status with elevated high-sensitivity c-reactive protein $(>8 \mathrm{mg} / \mathrm{L}$ ) or leukocytosis (white blood cell count $>$ 15,000 cells $\left./ \mathrm{mm}^{3} ; n=51\right)$, current malignancy $(n=54)$, 
refused or failed to jump ( $n=126)$, or any missing value in study variables were excluded from this analysis (Fig. 1).

\section{University of Wisconsin-Madison cohorts (United States Of America)}

Three hundred eighty-one individuals with weight-corrected jump power data from four different studies performed at the University of Wisconsin-Madison were included. Details about each study have been previously published [29, 34-36]. In brief, all studies recruited community-dwelling adults in the Midwestern United States (Wisconsin). All studies were cross-sectional and three of them only recruited older adults. In these three studies, exclusion criteria included recent (within the last 6 months) cardiovascular events, major surgery, severe end-organ disease, history of malignancy with metastasis to the musculoskeletal system, and neuromuscular disease impairing balance to the degree of being unable to stand without assistance. Less than $2 \%$ of participants who met these criteria were not able to perform a valid jump. In all studies, appendicular lean mass was measured by DXA using a GE/Lunar iDXA densitometer (Madison, WI, USA). Beside jump power, muscle function tests included grip strength and the short physical performance battery (SPPB) combining usual gait speed, 5-chair-rise-time, and balance assessment. All studies were approved by the local ethics committee. Participants younger than 65 years of age and those with missing data points were excluded (see Fig. 1).

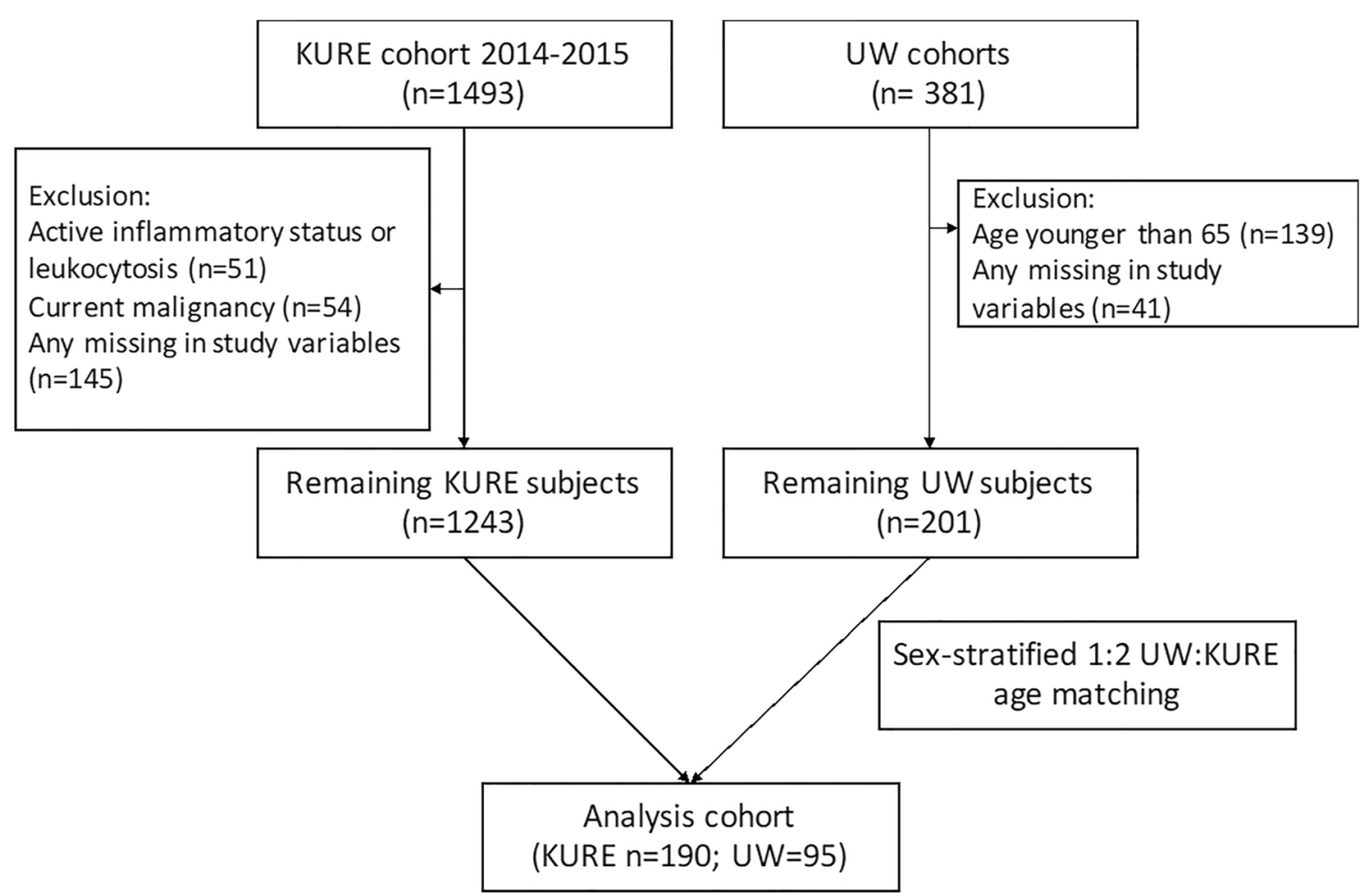

Fig. 1 Study flow. KURE, Korean Urban Rural Elderly cohort; UW, University of Wisconsin-Madison cohort 
As the initial step, individuals with low grip strength $(<27 \mathrm{~kg}$ in men and $<16 \mathrm{~kg}$ in women) or low five-times chair rise test performance ( $15 \mathrm{~s}$ or higher) were diagnosed as having "probable sarcopenia." Sarcopenia was "confirmed" when individuals with "probable sarcopenia" had low lean mass. Cut-offs for low lean mass in Caucasian and Asian cohorts were chosen based on regional normative populations according to the EWGSOP2 guideline. In the Caucasian cohort (UW), low lean mass was defined based on the normative reference from an Australian cohort (DXA-based ALM/height ${ }^{2}<7.0 \mathrm{~kg} / \mathrm{m}^{2}$ in men and $<5.5 \mathrm{~kg} / \mathrm{m}^{2}$ in women), which was the recommended cut-off by EWGSOP2 guideline for Europeans [3, 17, 39]. For the Asian cohort (KURE), low lean mass was defined by recommended cut-off from Asian Working Group for Sarcopenia guideline (BIA-based ALM/height ${ }^{2}<7.0 \mathrm{~kg} / \mathrm{m}^{2}$ in men; ALM/height ${ }^{2}<5.7 \mathrm{~kg} / \mathrm{m}^{2}$ in women) [40].

\section{Dysmobility syndrome}

In the Caucasian cohorts (UW), dysmobility syndrome was defined according to the criteria suggested by Binkley and colleagues [9]. The following six parameters were included in this dysmobility syndrome definition: history of one of more falls within last 12 months, slow gait speed $(<$ $1.0 \mathrm{~m} / \mathrm{s})$, low grip strength ( $<30 \mathrm{~kg}$ in men; $<20 \mathrm{~kg}$ in women), low appendicular lean mass (DXA-based ALM/height ${ }^{2}<$ $7.26 \mathrm{~kg} / \mathrm{m}^{2}$ in men; $<5.45 \mathrm{~kg} / \mathrm{m}^{2}$ in women), high fat mass (percent body fat $>30 \%$ in men; $>40 \%$ in women), and osteoporosis (BMD T score $\leq-2.5$ at lumbar spine, femoral neck, or total hip). Each included factor was equally weighted (1 point per risk factor), and the presence of three or more factors was defined as having dysmobility syndrome. In the Asian cohort (KURE), the same criteria were applied, with the exception of different cut-offs for low lean mass (BIA-based ALM $/$ height $^{2}<7.0 \mathrm{~kg} / \mathrm{m}^{2}$ in men; ALM $/$ height $^{2}<5.7 \mathrm{~kg} / \mathrm{m}^{2}$ in women) and low grip strength $(<26 \mathrm{~kg}$ in men; $<18 \mathrm{~kg}$ in women) based on regional reference endorsed by AWGS criteria [40].

\section{Composite outcome of sarcopenia or dysmobility syndrome}

The composite outcome of the presence of either sarcopenia or dysmobility syndrome was defined as the primary outcome to establish a practical cut-off for jump power that could be applied internationally.

\section{Statistics}

Data are presented as mean $\pm \mathrm{SD}$ or as numbers (percentages) as appropriate. Because KURE and UW cohorts showed significantly heterogeneous age distribution with higher mean age in UW (Supplementary Fig. 2A; $80.4 \pm 6.3$ vs. $71.4 \pm$ $4.3, p<0.001$ ), sex-stratified age matching with the range ( \pm
3 years) was performed with an 1:2 ratio (UW:KURE) to minimize the possibility of confounding from differences in age distribution in addition to differences in ethnicity. After matching, similar distribution of age was observed between two cohorts (Fig. 1; Supplementary Fig. 2B; mean age 74.9 \pm 3.9 vs. $75.3 \pm 3.7, p=0.373$ ). Clinical characteristics of participants in matched cohorts were compared using independent $t$ test for continuous variables or chi-squared tests for categorical variables. The relationship between weightcorrected jump power and age were plotted based on a prediction from linear regression as grouped by sex and ethnicity. Diagnostic cut-offs of low jump power yielding best binary classification for the composite outcome of sarcopenia (EWGSOP2) or dysmobility syndrome were estimated at the point value that maximizes the Liu index (sensitivity $\times$ specificity) on a receiver-operating characteristic (ROC) curve in groups stratified by the combination of sex and ethnicity [41]. As a sensitivity analysis, cut-offs were also estimated by Youden method as another ROC-based cut-off estimation algorithm (finding a cutoff that maximizes sensitivity + specificity -1). As cut-offs derived by both methods were largely dependent on sex but not by ethnicity, we decided to use identical cut-offs for KURE and UW participants within the same sex strata. Crude or adjusted odds ratios for sarcopenia, dysmobility syndrome, and the composite outcome of sarcopenia or dysmobility syndrome were calculated separately using multiple logistic regression models. Areas under the receiver-operating characteristics curve (AUC) were compared to quantify the improvement in discriminatory ability by low jump power when added to age, sex, height, and ethnicity. All analyses were performed by STATA 14.2 (College Station, TX, USA). The level of statistical significance was set at a two-sided $p<0.05$.

\section{Results}

\section{Study demographics}

Mean age ( $75.0 \pm 3.9$ years overall; 74.9 in KURE vs. 75.3 in $\mathrm{UW}, p=0.372)$ and proportion of women $(68.4 \%)$ did not differ between age-matched KURE and UW cohorts (Table 1). Individuals in the UW cohort had a higher mean BMI (26.7 vs. $\left.23.8 \mathrm{~kg} / \mathrm{m}^{2}, p<0.001\right)$. Weight-corrected jump power was similar between Caucasian and Asian cohorts in women and slightly higher in Asian cohort in men (KURE vs. $\mathrm{UW} ; 19.7$ vs. $18.6 \mathrm{~W} / \mathrm{kg}$ in women, $p=0.096 ; 26.9$ vs. $24.8 \mathrm{~W} / \mathrm{kg}$ in men, $p=0.050$ ). The prevalence of sarcopenia (EWGSOP2), dysmobility syndrome, and the composite outcome in combined cohorts were $7.4 \%, 29.1 \%$, and $30.9 \%$, respectively, and did not differ between UW and KURE (prevalence of sarcopenia $7.4 \%$ in both, dysmobility syndrome $31.6 \%$ and $27.9 \%$, and composite of either sarcopenia 
Table 1 Clinical characteristics of study participant

\begin{tabular}{|c|c|c|c|}
\hline & $\begin{array}{l}\text { KURE } \\
(\mathrm{n}=190)\end{array}$ & $\begin{array}{l}\text { UW } \\
(n=95)\end{array}$ & $\begin{array}{l}p \\
\text { value }\end{array}$ \\
\hline Age, years & $74.9 \pm 3.9$ & $75.3 \pm 3.7$ & 0.372 \\
\hline Women, n (\%) & $130(68.4)$ & $65(68.4)$ & 0.999 \\
\hline BMI, $\mathrm{kg} / \mathrm{m}^{2}$ & $23.8 \pm 3.1$ & $26.7 \pm 4.9$ & $<0.001$ \\
\hline Maximum jump height, $\mathrm{cm}$ & $17.9 \pm 8.6$ & $18.4 \pm 6.7$ & 0.628 \\
\hline Weight-corrected jump power, W/kg (men) & $26.9 \pm 4.7$ & $24.8 \pm 4.6$ & 0.050 \\
\hline Weight-corrected jump power, W/kg (women) & $19.7 \pm 4.5$ & $18.6 \pm 4.6$ & 0.096 \\
\hline Sarcopenia (EWGSOP2), n (\%) & $14(7.4)$ & $7(7.4)$ & 0.999 \\
\hline Dysmobility syndrome, n (\%) & $53(27.9)$ & $30(31.6)$ & 0.519 \\
\hline $\begin{array}{l}\text { Composite outcome (presence of either sarcopenia or dysmobility } \\
\text { syndrome), } \mathrm{n}(\%)\end{array}$ & $54(28.4)$ & $31(32.6)$ & 0.650 \\
\hline Low grip strengtha, $\mathrm{n}(\%)$ & $20(10.5)$ & $17(17.9)$ & 0.081 \\
\hline Slow gait speed ${ }^{\mathrm{b}}, \mathrm{n}(\%)$ & $83(43.7)$ & $18(18.9)$ & $<0.001$ \\
\hline High fat mass ${ }^{\mathrm{c}}, \mathrm{n}(\%)$ & $37(19.5)$ & $44(46.3)$ & $<0.001$ \\
\hline Low lean mass ${ }^{\mathrm{d}}, \mathrm{n}(\%)$ & $49(25.8)$ & $19(18.9)$ & 0.199 \\
\hline Falls in previous year, $\mathrm{n}(\%)$ & $51(26.8)$ & $33(34.7)$ & 0.168 \\
\hline Osteoporosis ${ }^{\mathrm{e}}, \mathrm{n}(\%)$ & $80(42.1)$ & $25(26.3)$ & 0.009 \\
\hline
\end{tabular}

Abbreviations: KURE, Korean Urban Rural Elderly cohort (Koreans); UW, University of Wisconsin-Madison cohort (Caucasians); AWGS, Asian Working Group for Sarcopenia; EWGSOP2, revised sarcopenia definition by European Working Group on Sarcopenia in Older People

${ }^{\text {a }}$ Grip strength $<27 \mathrm{~kg}$ in men and $<16 \mathrm{~kg}$ in women according to EWGSOP2 sarcopenia definition

${ }^{\mathrm{b}}$ Gait speed $<1.0 \mathrm{~m} / \mathrm{s}$

${ }^{\mathrm{c}}$ Percent body fat $>30 \%$ in men and $>40 \%$ in women

${ }^{\mathrm{d}}$ Dual-energy x-ray absorptiometry (DXA)-based appendicular lean mass (ALM)/height ${ }^{2}<7.26 \mathrm{~kg} / \mathrm{m}^{2}$ (men) and $<5.45 \mathrm{~kg} / \mathrm{m}^{2}$ women in the UW cohort according to the dysmobility syndrome definition; BIA-based ALM/ height ${ }^{2}<7.0 \mathrm{~kg} / \mathrm{m}^{2}$ (men) and $<5.7 \mathrm{~kg} / \mathrm{m}^{2}$ women in the KURE cohort according to the Asian Working Group for Sarcopenia definition

${ }^{\mathrm{e}}$ Bone mineral density T-score -2.5 or lower at lumbar spine, femoral neck, or total hip measured by DXA or dysmobility syndrome $32.6 \%$ and $30.0 \%$, respectively). Among the six components of dysmobility syndrome, UW participants were more likely to have higher prevalence of high fat mass compared with KURE participants, whereas KURE participants had higher prevalence of slow gait speed and osteoporosis (Table 1). Weight-corrected jump power showed weak correlations with grip strength $(r=0.37$ and 0.33 in men and women, respectively; Supplementary Fig. 3).

\section{Relationship of jump power and age}

There was a negative linear correlation between weightcorrected jump power and age in both men and women (Supplementary Fig. 4). Per 5-year increment in age, weightcorrected jump power was reduced by $2.0 \mathrm{~W} / \mathrm{kg}(95 \% \mathrm{CI} 3.4$ to $-0.6, p=0.005)$ and by $1.5 \mathrm{~W} / \mathrm{kg}(95 \% \mathrm{CI} 2.5$ to $-0.5, p=$ 0.003 ) in men and women, respectively. No significant interaction between age and ethnicity was observed ( $p$ for interaction $=0.915$ in men; $p$ for interaction $=0.201$ in women). When vertical jump power was normalized by a lean mass index (appendicular lean mass $/$ height $^{2}, \mathrm{~W} / \mathrm{kg} / \mathrm{m}^{2}$ ), the fitted values for age of the two cohorts aligned more closely and without a significant difference in mean values (Supplementary Fig. 5; UW vs. KURE: 265 vs. 269 W/kg/ $\mathrm{m}^{2}$ in men, $p=0.733 ; 202$ vs. $206 \mathrm{~W} / \mathrm{kg} / \mathrm{m}^{2}$ in women, $p=$ 0.609).

\section{Jump power cut-off for the composite outcome}

The sex-specific weight-corrected jump power cut-offs for the presence of either sarcopenia or dysmobility syndrome (the composite outcome) were estimated to find the point value that maximizes the binary discriminatory performance for each sex and ethnic group combination (Table 2). Identified optimal cut-offs to detect the composite outcome largely depended on sex but not ethnicity for UW and KURE participants $(<19.0 \mathrm{~W} / \mathrm{kg}$ and $<19.1 \mathrm{~W} / \mathrm{kg}$ for UW and KURE women; $<22.6 \mathrm{~W} / \mathrm{kg}$ and $<24.7 \mathrm{~W} / \mathrm{kg}$ for UW and KURE men). When Caucasian and Asian cohorts were combined, the optimal binary cut-off for low weight-corrected jump power was set at $<19.0 \mathrm{~W} / \mathrm{kg}$ (sensitivity 0.72 , specificity 0.67 , AUC at point 0.69 ) in women and $<23.8 \mathrm{~W} / \mathrm{kg}$ (sensitivity 
Table 2 Proposed cut-offs of jump power for identifying individuals with composite outcome as presence of either sarcopenia (EWGSOP2) or dysmobility syndrome

\begin{tabular}{lllll}
\hline Groups & Cut-off*, W/kg & Sensitivity & Specificity & AUC at point \\
\hline UW women $(n=65)$ & $<19.0$ & 0.81 & 0.74 & 0.78 \\
UW men $(n=30)$ & $<22.6$ & 0.75 & 0.73 & 0.74 \\
KURE women $(n=130)$ & $<19.1$ & 0.66 & 0.65 & 0.66 \\
KURE men $(n=60)$ & $<24.7$ & 0.86 & 0.79 & 0.82 \\
Women (UW and KURE; $n=195)$ & $<19.0$ & 0.72 & 0.67 & 0.69 \\
Men (UW and KURE; $n=90)$ & $<23.8$ & 0.73 & 0.81 & 0.77 \\
\hline
\end{tabular}

*Diagnostic cut-offs were determined using Liu methods in each group. Identical results were obtained when Youden index was applied to find cut-offs in each group

KURE, Korean Urban Rural Elderly cohort (Koreans); UW, University of Wisconsin-Madison cohort (Caucasians); $A U C$, area under the receiver-operating characteristics curve
0.73 , specificity $0.81, \mathrm{AUC}$ at point 0.77 ) in men. The sensitivity and specificity of the cut-offs in overall participants were 0.68 and 0.73 . When the predicted probability of the composite outcome estimated from logistic regression model (y axis) was plotted against weight-corrected jump power ( $\mathrm{x}$ axis), the proposed cut-offs aligned near the inflection point for the steeper increase of probability of the presence of composite outcome in UW and KURE men and women (Fig. 2). Similar results were observed for sarcopenia alone or dysmobility syndrome alone as outcomes (Supplementary Fig. 6).

\section{Odds ratio of sarcopenia or dysmobility syndrome according to low jump power}

Participants with low weight-corrected jump power (<19.0 W/ $\mathrm{kg}$ in women; $<23.8 \mathrm{~W} / \mathrm{kg}$ in men) had 5.8-fold elevated odds of having either sarcopenia or dysmobility syndrome (composite endpoint) compared with those with normal to high jump power (crude odds ratio [OR] 5.80, 95\% CI 3.34-10.09, $p<0.001$ ). The association between low jump power and the composite of sarcopenia or dysmobility syndrome remained robust after adjustment for age, sex, height, and ethnic groups (UW vs. KURE)( adjusted OR [aOR] 4.67, 95\% CI 2.60-8.38, $p<0.001$ ), with similar results for sarcopenia (aOR 4.07, 95\% CI 1.45-11.41, $p=0.008$ ) alone or dysmobility syndrome alone (aOR 4.32, 95\% CI 2.40-7.80, $p<0.001$ ). See Table 3. When weightcorrected jump power was entered into the multiple logistic model as a continuous variable, $1-\mathrm{W} / \mathrm{kg}$ decrease of jump power was associated with $20 \%$ elevated odds of presence of sarcopenia or dysmobility syndrome (composite outcome) independent of covariates (aOR 1.20, 95\% CI 1.11-1.28, $p<0.001$ ).

\section{Additive discriminatory value of low jump power for sarcopenia or dysmobility syndrome}

In ROC analyses, weight-corrected jump power alone yielded modest discriminatory performance for the composite of sarcopenia or dysmobility syndrome (AUC 0.77), which was comparable with that of a model including age, sex, height, and ethnic groups (model 1 in Fig. 3; AUC 0.72 vs. 0.77, $p=0.123$ ). Jump height yielded a similar AUC to jump power (Supplementary Fig. 7, AUC 0.76 vs. $0.77, p=0.658$ ). Adding low jump power to model 1 significantly improved discriminatory performance for the composite of sarcopenia or dysmobility syndrome (AUC 0.72 to $0.79, p=0.004)$.

\section{Discussion}

This analysis of age- and sex-matched Asian and Caucasian older adults indicates that jump power values of $<19.0 \mathrm{~W} / \mathrm{kg}$ in women and $<23.8 \mathrm{~W} / \mathrm{kg}$ in men can be utilized as an international threshold for identifying individuals with sarcopenia or dysmobility syndrome. Low jump power defined by these cutoffs was an independent risk factor for the composite outcome with an odds ratio of 4.67 , which improved the discrimination for individuals with sarcopenia or dysmobility syndrome when considered together with age, sex, height, and ethnicity.

These findings are well aligned with previous reports showing that jump power improves discrimination between those with or without dysmobility syndrome in the KURE cohort [24]. In an analysis of US cohorts, Siglinsky and colleagues proposed jump power cut-offs to be in the range of $15-17 \mathrm{~W} / \mathrm{kg}$ depending on which sarcopenia definition was used. Those without sarcopenia had values of approximately $20 \mathrm{~W} / \mathrm{kg}$ [30]. However, cutoffs were not separated by sex. Singh and colleagues also found that individuals with sarcopenia had lower jump power compared with those without, even though they used a different method for measuring jump power [31]. Intriguingly, the distribution of jump power in these various cohorts was similar to the range of jump power in our study. [19-21, 23-25, 28-31, 34, 35]. Although 
Fig. 2 Predicted probability of the composite outcome, defined as presence of either sarcopenia or dysmobility syndrome, according to weight-corrected jump power. Sarcopenia was defined using EWGSOP2 guideline. Probability of composite outcome was estimated from logistic regression model, which included weightcorrected jump power, cohort types, and the interaction term between two variables as independent variables. *Composite outcome: presence of either sarcopenia or dysmobility syndrome
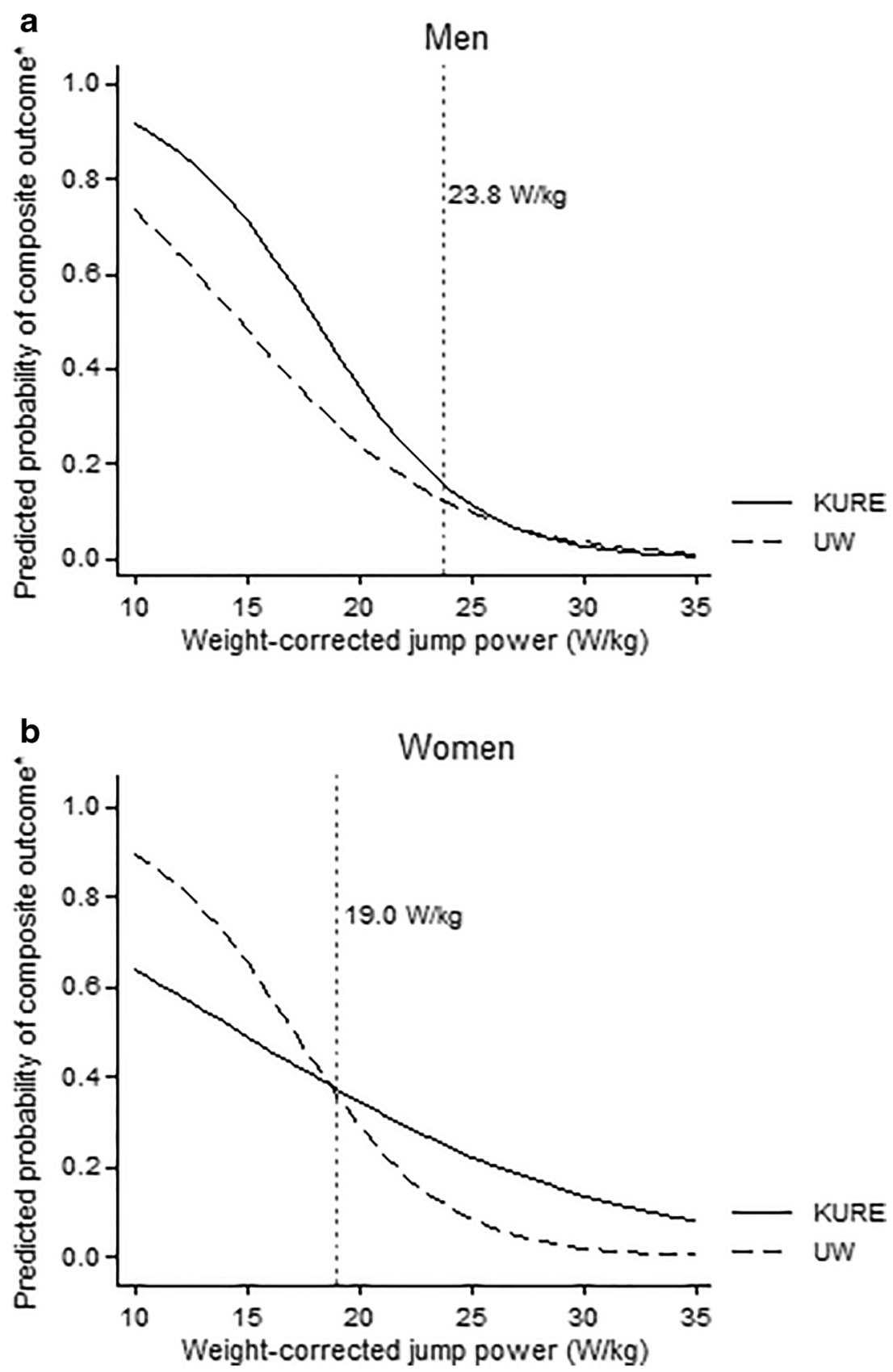

participants were not separated into those with sarcopenia or without in these studies, the similarity in the range of jump power suggests that our results might be transferable to other populations. However, the proposed cut-offs need to be validated in additional cohorts and ethnicities other than Asian and Caucasian.
Table 3 Association of low jump power defined by proposed international threshold with sarcopenia, dysmobility syndrome, or the composite of sarcopenia or dysmobility syndrome in communitydwelling older adults

\begin{tabular}{llrlr}
\hline Outcomes & Crude OR $(95 \% \mathrm{CI})$ & $p$ value & Adjusted OR (95\% CI)* & $p$ value \\
\hline Composite outcome $\dagger$ & $5.80(3.34-10.09)$ & $<0.001$ & $4.67(2.60-8.38)$ & $<0.001$ \\
$\begin{array}{l}\text { Sarcopenia (by EWGSOP2 } \\
\text { definition) }\end{array}$ & $4.30(1.61-11.45)$ & 0.003 & $4.07(1.45-11.41)$ & 0.008 \\
\begin{tabular}{l} 
Dysmobility syndrome \\
\hline
\end{tabular} & $5.41(3.11-9.40)$ & $<0.001$ & $4.32(2.40-7.80)$ & $<0.001$ \\
\hline
\end{tabular}

*Adjusted for age, sex, height, and ethnicity (UW vs. KURE)

$\dagger$ Composite outcome: either presence of sarcopenia (EWGSOP2) or dysmobility syndrome 
Fig. 3 Improved discriminatory performance for the composite outcome when low jump power was added to model 1 including age, sex, height, and ethnicity. The composite outcome was defined as the presence of either sarcopenia or dysmobility syndrome. Discriminatory performance of weight-corrected jump power alone was similar to those of model 1

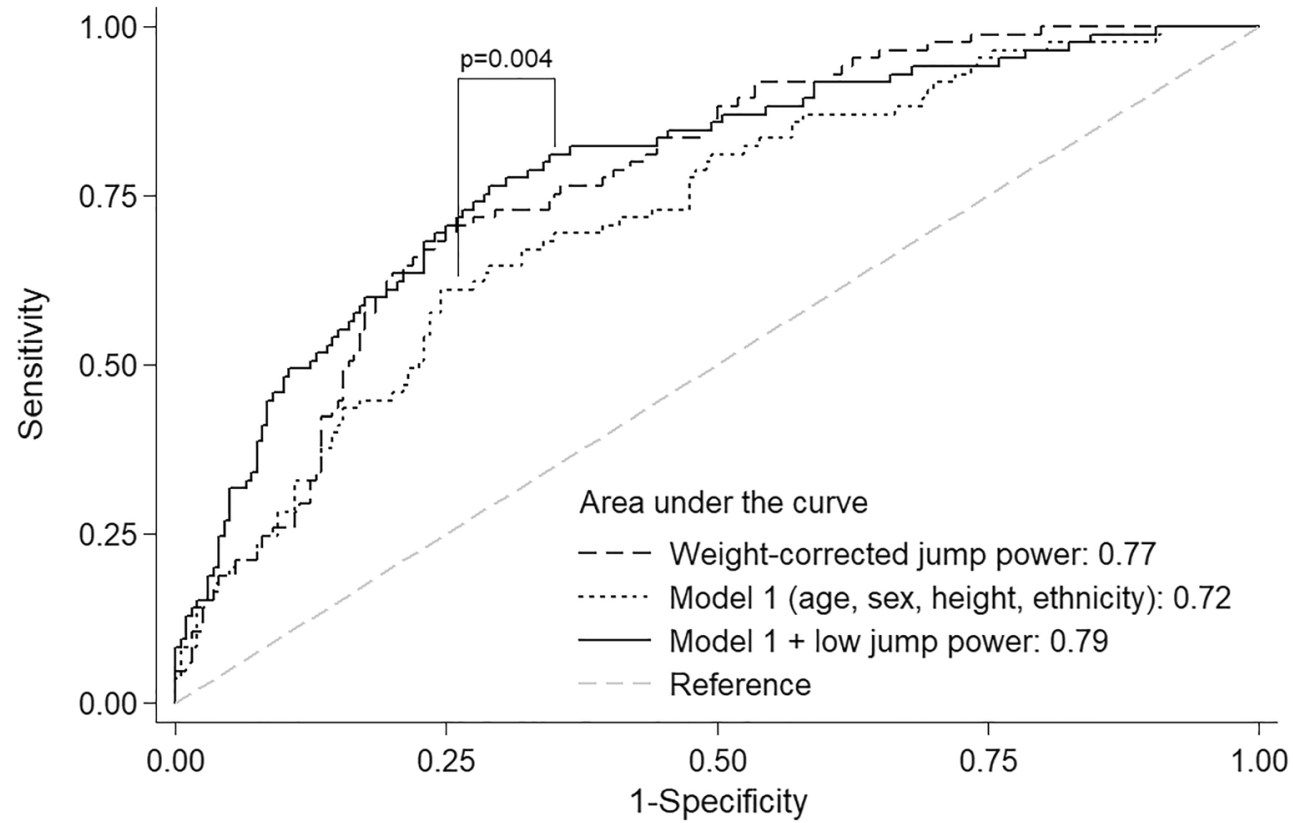

As there is no agreement on one single sarcopenia definition and several cut-offs for various muscle and physical function tests exist, we deliberately chose the establishment of cutoffs for jump power that could be generalizable not only internationally to different populations but also for individuals with or without any sarcopenia or dysmobility syndrome as the primary aim of this study. To do so, we decided that our endpoint would combine different ways to define poor musculoskeletal health. First, the sarcopenia was defined according to EWGSOP2 algorithm. It is important to note that the recent 2018 EWGSOP2 definition differs significantly from the 2010 EWGSOP definition leading to different sarcopenia prevalence in the same population [7]. In our view, the 2018 EWGSOP2 definition has clear advantages because "probable sarcopenia" can now be diagnosed without measuring lean mass, a clinically very important change. The previous limitation to depend on lean mass as a parameter to define poor musculoskeletal health but not on function was also one reason for the development of dysmobility syndrome. In dysmobility syndrome, low lean mass is only one of six equally weighted factors included in the definition. Dysmobility syndrome not only balances the importance of low lean mass but it also includes other organ systems that play a vital role in musculoskeletal health, namely, the bone, fat, and indirectly (through the prevalence of falls) the nervous system [9-12, 42]. The criteria and cut-offs were chosen based on a thorough literature review but these need to be adjusted as more data becomes available [9-12, 24, 42, 43]. Because dysmobility syndrome approaches musculoskeletal health differently, we felt that using a composite outcome of either dysmobility syndrome or sarcopenia in two cohorts with different ethnicities would increase the likelihood of finding cut-offs that can be applied to different populations and different definitions of musculoskeletal health. Using such a composite outcome is further supported by the fact that the association with decreased mobility and increased falls, fractures, and mortality is similar between the two entities [1, 3, 5, 7, 12-16, 42, 44]. Other authors have used other terms and parameters to describe syndromes of impaired muscle, low bone mass, and obesity, including osteosarcopenic obesity or locomotive syndrome [43-45]. Future studies are needed to examine whether the proposed jump power cut-offs can also be applied to these syndromes.

The jump power measurement has advantages as a test with good reproducibility, good correlation with other methods to measure physical function and muscle mass, little or no ceiling effect, and good safety [4, 20, 21, 28, 29]. Relatively weak correlations between hand grip strength and jump power shown in prior studies may indicate that jump power has an advantage over hand grip strength as a parameter for lower extremity function, a clinically relevant region due to the association with falls and hip fractures in older adults [46]. However, some limitations need to be pointed out. Jump power is measured on a force plate, which limits the portability of the method with additional cost. However, research is ongoing whether jump power can be assessed without using a force plate $[31,47,48]$. Another limitation is the current scarcity of studies that have prospectively examined the direct relationship of low jump power with health outcomes such as falls, fractures, immobility, decreased daily activity, or mortality, although cross-sectional studies robustly support the association between lower jump power or inability to jump with worse ADLs and more falls [23, 28]. Some large, wellestablished musculoskeletal cohorts, such as the MrOS study, 
have included jump power in their assessment recently, and prospective data should be available in the near future. Our hope is that the proposition of jump power cut-offs will encourage other groups to start using jump power in their research to validate our results and examine the relationship of jump power and health outcomes. In our prior work, we found that individuals who failed or refused to jump due to various reasons had elevated odds of dysmobility syndrome in community-dwelling Korean older adults [24]. Based on these findings, it would be reasonable to consider groups who had jump power lower than the identified thresholds or those who failed (or refused) to jump as high risk groups. This needs to be validated in further studies.

Creating an age- and gender-matched dataset out of two cohorts of community-dwelling older adults from two different ethnic backgrounds enables us to reduce confounding from variables other than ethnicity but also led to a smaller sample size mainly due to the age gap between two cohorts. As already pointed out, this study did not include other ethnicities, and as such, further studies are necessary to show that these cut-offs are truly "international." However, we feel that it is a clear strength to have examined individuals from the two large ethnic groups, Caucasian and Asian. Because there currently is no consensus of the optimal cut-off for low lean mass among different ethnicities and the methodology for measuring lean mass was different in two ethnic cohorts, regional definitions were applied per EWGSOP2 by race and the ALM acquisition methodology used to derive respective cutoffs $[3,35,39,40]$. We used the DXA-based EWGSOP 2018 appendicular lean mass cut-offs for the Caucasian (UW) cohorts and the BIA-based AWGS cut-offs to define low lean mass for the Asian cohort (KURE) based on Asian normative references $[3,40]$. Despite the differences in lean mass assessment between two cohorts, we observed similar prevalence of sarcopenia, dysmobility syndrome, and composite outcome between age-matched Caucasian and Asian cohorts in this study. Another point that needs to be noted is the differences in body composition between the Korean-Asian and USCaucasian population. High fat mass was more prevalent in the Caucasian individuals, whereas low lean mass was more common in the Asian cohort. However, the distribution of their muscle function, particularly muscle power, was similar regardless of the differences in body composition. To us, this is an advantage of a score-based diagnosis rather than an approach where the diagnosis of sarcopenia solely relies on the presence of low lean mass. Which factors should be included in such a score-based approach to diagnose poor musculoskeletal health is still up for discussion. The dysmobility syndrome approach is a simple and powerful but can certainly be improved further. The results from this study suggest that one possibility would be the inclusion of jump power in the definition of dysmobility syndrome or sarcopenia, which merits further validation.

\section{Conclusion}

The results of this combined analysis of older communitydwelling adults of Asian and Caucasian ethnicities indicate that it is possible to use sex-stratified jump power cut-offs to discriminate between individuals with either sarcopenia or dysmobility syndrome and without. Low jump power defined by the proposed cut-off of $19.0 \mathrm{~W} / \mathrm{kg}$ for females and $23.8 \mathrm{~W} /$ $\mathrm{kg}$ for males led to 4.7-fold elevated odds for the composite outcome of either sarcopenia or dysmobility syndrome with improved discriminatory performance, independent of age, sex, height, and ethnicity. Further studies are needed to validate the proposed international cut-offs in other ethnicities with predictive value for falls, fractures, and mortality.

Funding This research was funded by Research of Korea Centers for Disease Control and Prevention (2012-E63001-001, 2013-E63007-00, 2013-E63007-01, 2013-E63007-02, 2013-ER6302-00). Open Access funding provided by Projekt DEAL.

\section{Compliance with ethical standards}

Conflicts of interest Dr. Buehring reports grants from MSD, Kinemed and GE/Lunar related to the current work. Additionally, he reports grants and personal fees from Lilly, grants from Extendicare Foundation, personal fees from GE Healthcare, personal fees from UCB, Janssen, Gilead and AbbVie outside the submitted work. Ms. Krueger reports research grants from Radius and personal fees from Amgen outside the submitted work. Dr. Binkley reports grants from Radius, RTI Health Solutions, and GE Healthcare and personal fees from Amgen and Taurus, all outside of the submitted work. Dr. Rhee reports personal fees from Amgen outside of the submitted work. All other authors report no conflict of interest.

Open Access This article is licensed under a Creative Commons Attribution-NonCommercial 4.0 International License, which permits any non-commercial use, sharing, adaptation, distribution and reproduction in any medium or format, as long as you give appropriate credit to the original author(s) and the source, provide a link to the Creative Commons licence, and indicate if changes were made. The images or other third party material in this article are included in the article's Creative Commons licence, unless indicated otherwise in a credit line to the material. If material is not included in the article's Creative Commons licence and your intended use is not permitted by statutory regulation or exceeds the permitted use, you will need to obtain permission directly from the copyright holder. To view a copy of this licence, visit http://creativecommons.org/licenses/by-nc/4.0/.

\section{References}

1. Beaudart C, Zaaria M, Pasleau F, Reginster JY, Bruyere O (2017) Health outcomes of sarcopenia: a systematic review and meta-analysis. PLoS One 12(1):e0169548. https://doi.org/10.1371/journal. pone. 0169548

2. Bruyere O, Beaudart C, Ethgen O, Reginster JY, Locquet M (2019) The health economics burden of sarcopenia: a systematic review. Maturitas 119:61-69. https://doi.org/10.1016/j.maturitas.2018.11. 003 
3. Cruz-Jentoft AJ, Bahat G, Bauer J, Boirie Y, Bruyère O, Cederholm T, Cooper C, Landi F, Rolland Y, Sayer AA, Schneider SM, Sieber CC, Topinkova E, Vandewoude M, Visser M, Zamboni M, Writing Group for the European Working Group on Sarcopenia in Older People 2 (EWGSOP2), and the Extended Group for EWGSOP2 (2019) Sarcopenia: revised European consensus on definition and diagnosis. Age Ageing 48:601. https://doi.org/10.1093/ageing/ afz046

4. Edwards MH, Buehring B (2015) Novel approaches to the diagnosis of sarcopenia. J Clin Densitom 18:472-477. https://doi.org/10. 1016/j.jocd.2015.04.010

5. Wong RMY, Wong H, Zhang N, Chow SKH, Chau WW, Wang J, Chim YN, Leung KS, Cheung WH (2019) The relationship between sarcopenia and fragility fracture-a systematic review. Osteoporos Int 30(3):541-553. https://doi.org/10.1007/s00198018-04828-0

6. Cawthon PM, Travison TG, Manini TM et al (2019) Establishing the link between lean mass and grip strength cut-points with mobility disability and other health Outcomes: Proceedings of the Sarcopenia Definition and Outcomes Consortium Conference. J Gerontol A Biol Sci Med Sci. https://doi.org/10.1093/gerona/ glz081

7. Locquet M, Beaudart C, Petermans J, Reginster JY, Bruyere O (2019) EWGSOP2 versus EWGSOP1: impact on the prevalence of sarcopenia and its major health consequences. J Am Med Dir Assoc 20(3):384-385. https://doi.org/10.1016/j.jamda.2018.11.027

8. Menant JC, Weber F, Lo J, Sturnieks DL, Close JC, Sachdev PS, Brodaty H, Lord SR (2017) Strength measures are better than muscle mass measures in predicting health-related outcomes in older people: time to abandon the term sarcopenia? Osteoporos Int 28(1): 59-70. https://doi.org/10.1007/s00198-016-3691-7

9. Binkley N, Krueger D, Buehring B (2013) What's in a name revisited: should osteoporosis and sarcopenia be considered components of "dysmobility syndrome?". Osteoporos Int 24(12):29552959. https://doi.org/10.1007/s00198-013-2427-1

10. Lewiecki EM, Bilezikian JP, Binkley N, Hans D, Krueger D, Miller PD, Oates M, Shane E (2015) Update on osteoporosis from the 2014 Santa Fe Bone symposium. Endocr Res 40(2):106-119. https://doi.org/10.3109/07435800.2015.1005746

11. Binkley N, Blank RD, Leslie WD, Lewiecki EM, Eisman JA, Bilezikian JP (2017) Osteoporosis in crisis: it's time to focus on fracture. J Bone Miner Res 32(7):1391-1394. https://doi.org/10. $1002 / \mathrm{jbmr} .3182$

12. Buehring B, Hansen KE, Lewis BL, Cummings SR, Lane NE, Binkley N, Ensrud KE, Cawthon PM (2018) Dysmobility syndrome independently increases fracture risk in the osteoporotic fractures in men (MrOS) prospective cohort study. J Bone Miner Res 33(9):1622-1629. https://doi.org/10.1002/jbmr.3455

13. Iolascon G, Moretti A, Giamattei MT, Migliaccio S, Gimigliano F (2015) Prevalent fragility fractures as risk factor for skeletal muscle function deficit and dysmobility syndrome in post-menopausal women. Aging Clin Exp Res 27(Suppl 1):S11-S16. https://doi. org/10.1007/s40520-015-0417-1

14. Lee WJ, Liu LK, Hwang AC, Peng LN, Lin MH, Chen LK (2017) Dysmobility syndrome and risk of mortality for communitydwelling middle-aged and older adults: the nexus of aging and body composition. Sci Rep 7(1):8785. https://doi.org/10.1038/s41598017-09366-Z

15. Looker AC (2014) Dysmobility syndrome and mortality risk in US men and women age 50 years and older. Osteoporos Int 26:93-102. https://doi.org/10.1007/s00198-014-2904-1

16. Clynes MA, Edwards MH, Buehring B, Dennison EM, Binkley N, Cooper C (2015) Definitions of sarcopenia: associations with previous falls and fracture in a population sample. Calcif Tissue Int 97(5):445-452. https://doi.org/10.1007/s00223-015-0044-z
17. Studenski SA, Peters KW, Alley DE, Cawthon PM, McLean RR, Harris TB, Ferrucci L, Guralnik JM, Fragala MS, Kenny AM, Kiel DP, Kritchevsky SB, Shardell MD, Dam TTL, Vassileva MT (2014) The FNIH sarcopenia project: rationale, study description, conference recommendations, and final estimates. J Gerontol Ser A-Biol Sci Med Sci 69(5):547-558. https://doi.org/10.1093/gerona/ glu010

18. Cawthon PM (2015) Assessment of lean mass and physical performance in sarcopenia. J Clin Densitom 18(4):467-471. https://doi. org/10.1016/j.jocd.2015.05.063

19. Buehring B, Krueger D, Binkley N (2010) Jumping mechanography: a potential tool for sarcopenia evaluation in older individuals. J Clin Densitom 13(3):283-291. https://doi.org/10. 1016/j.jocd.2010.04.002

20. Taani MH, Kovach CR, Buehring B (2017) Muscle Mechanography: a novel method to measure muscle function in older adults. Res Gerontol Nurs 10(1):17-24. https://doi.org/10. 3928/19404921-20161209-03

21. Rittweger J, Schiessl H, Felsenberg D, Runge M (2004) Reproducibility of the jumping mechanography as a test of mechanical power output in physically competent adult and elderly subjects. J Am Geriatr Soc 52(1):128-131

22. Veilleux LN, Rauch F (2010) Reproducibility of jumping mechanography in healthy children and adults. J Musculoskelet Neuronal Interact 10(4):256-266

23. Strotmeyer ES, Winger ME, Cauley JA et al (2018) Normative values of muscle power using force plate jump tests in men aged 77-101 years: the osteoporotic fractures in men (MrOS) study. J Nutr Health Aging 22(10):1167-1175. https://doi.org/10.1007/ s12603-018-1081-x

24. Hong N, Kim CO, Youm Y, Kim HC, Rhee Y (2018) Low peak jump power is associated with elevated odds of dysmobility syndrome in community-dwelling elderly individuals: the Korean Urban Rural Elderly (KURE) study. Osteoporos Int 29(6):14271436. https://doi.org/10.1007/s00198-018-4466-0

25. Stolzenberg N, Felsenberg D, Belavy DL (2018) Postural control is associated with muscle power in post-menopausal women with low bone mass. Osteoporos Int 29(10):2283-2288. https://doi.org/10. 1007/s00198-018-4599-1

26. Michaelis I, Kwiet A, Gast U, Boshof A, Antvorskov T, Jung T, Rittweger J, Felsenberg D (2008) Decline of specific peak jumping power with age in master runners. J Musculoskelet Neuronal Interact 8(1):64-70

27. Ward KA, Das G, Berry JL, Roberts SA, Rawer R, Adams JE, Mughal Z (2009) Vitamin D status and muscle function in postmenarchal adolescent girls. J Clin Endocrinol Metab 94(2):559563. https://doi.org/10.1210/jc.2008-1284

28. Winger ME, Caserotti P, Cauley JA et al (2019) Associations between novel jump test measures, grip strength, and physical performance: the osteoporotic fractures in men (MrOS) study. Aging Clin Exp Res 32:587-595. https://doi.org/10.1007/s40520-019-01421-1

29. Buehring B, Krueger D, Fidler E, Gangnon R, Heiderscheit B, Binkley N (2015) Reproducibility of jumping mechanography and traditional measures of physical and muscle function in older adults. Osteoporos Int 26(2):819-825. https://doi.org/10.1007/ s00198-014-2983-z

30. Siglinsky E, Krueger D, Ward RE, Caserotti P, Strotmeyer ES, Harris TB, Binkley N, Buehring B (2015) Effect of age and sex on jumping mechanography and other measures of muscle mass and function. J Musculoskelet Neuronal Interact 15(4):301-308

31. Singh H, Kim D, Kim E, Bemben MG, Anderson M, Seo DI, Bemben DA (2014) Jump test performance and sarcopenia status in men and women, 55 to 75 years of age. J Geriatr Phys Ther 37(2):76-82. https://doi.org/10.1519/JPT.0b013e3182a51b11

32. Hong N, Kim KJ, Lee SJ, Kim CO, Kim HC, Rhee Y, Youm Y, Choi JY, Park HY (2019) Cohort profile: Korean urban rural elderly 
(KURE) study, a prospective cohort on ageing and health in Korea. BMJ Open 9(10):e031018. https://doi.org/10.1136/bmjopen-2019031018

33. Lee EY, Kim HC, Rhee Y, Youm Y, Kim KM, Lee JM, Choi DP, Yun YM, Kim CO (2014) The Korean urban rural elderly cohort study: study design and protocol. BMC Geriatr 14:33. https://doi. org/10.1186/1471-2318-14-33

34. Buehring B, Hind J, Fidler E, Krueger D, Binkley N, Robbins J (2013) Tongue strength is associated with jumping mechanography performance and handgrip strength but not with classic functional tests in older adults. J Am Geriatr Soc 61(3):418-422. https://doi. org/10.1111/jgs. 12124

35. Buehring B, Siglinsky E, Krueger D, Evans W, Hellerstein M, Yamada Y, Binkley N (2018) Comparison of muscle/lean mass measurement methods: correlation with functional and biochemical testing. Osteoporos Int 29(3):675-683. https://doi.org/10.1007/ s00198-017-4315-6

36. Radler BT (2014) The midlife in the United States (MIDUS) series: a national longitudinal study of health and well-being. Open Health Data 2(1). https://doi.org/10.5334/ohd.ai

37. Buehring B, Krueger D, Binkley N (2010) Jumping mechanography: a potential tool for sarcopenia evaluation in older individuals. J Clin Densitom 13(3):283-291. https://doi.org/10. 1016/j.jocd.2010.04.002

38. Buehring B, Krueger D, Fidler E, Gangnon R, Heiderscheit B, Binkley N (2015) Reproducibility of jumping mechanography and traditional measures of physical and muscle function in older adults. Osteoporos Int 26(2):819-825. https://doi.org/10.1007/ s00198-014-2983-z

39. Gould H, Brennan SL, Kotowicz MA, Nicholson GC, Pasco JA (2014) Total and appendicular lean mass reference ranges for Australian men and women: the Geelong osteoporosis study. Calcif Tissue Int 94(4):363-372. https://doi.org/10.1007/s00223013-9830-7

40. Chen LK, Liu LK, Woo J, Assantachai P, Auyeung TW, Bahyah KS, Chou MY, Chen LY, Hsu PS, Krairit O, Lee JSW, Lee WJ, Lee Y, Liang CK, Limpawattana P, Lin CS, Peng LN, Satake S, Suzuki T, Won CW, Wu CH, Wu SN, Zhang T, Zeng P, Akishita M, Arai $\mathrm{H}$ (2014) Sarcopenia in Asia: consensus report of the Asian Working Group for Sarcopenia. J Am Med Dir Assoc 15(2):95101. https://doi.org/10.1016/j.jamda.2013.11.025
41. Liu X (2012) Classification accuracy and cut point selection. Stat Med 31(23):2676-2686. https://doi.org/10.1002/sim.4509

42. Hong N, Kim CO, Youm Y, Choi JY, Kim HC, Rhee Y (2018) Dysmobility syndrome is associated with prevalent morphometric vertebral fracture in older adults: the Korean Urban-Rural Elderly (KURE) study. Arch Osteoporos 13(1):86. https://doi.org/10.1007/ s11657-018-0500-2

43. Yi HS, Lee S (2018) Overcoming osteoporosis and beyond: locomotive syndrome or dysmobility syndrome. Osteoporos Sarcopenia 4(3):77-78. https://doi.org/10.1016/j.afos.2018.09.001

44. Huo YR, Suriyaarachchi P, Gomez F, Curcio CL, Boersma D, Gunawardene P, Demontiero O, Duque G (2016) Phenotype of sarcopenic obesity in older individuals with a history of falling. Arch Gerontol Geriatr 65:255-259. https://doi.org/10.1016/j. archger.2016.04.003

45. Bauer JM, Cruz-Jentoft AJ, Fielding RA, Kanis JA, Reginster JY, Bruyère $\mathrm{O}$, Cesari $\mathrm{M}$, Chapurlat $\mathrm{R}$, al-Daghri $\mathrm{N}$, Dennison $\mathrm{E}$, Kaufman JM, Landi F, Laslop A, Locquet M, Maggi S, McCloskey E, Perna S, Rizzoli R, Rolland Y, Rondanelli M, Szulc P, Vellas B, Vlaskovska M, Cooper C (2019) Is there enough evidence for osteosarcopenic obesity as a distinct entity? A critical literature review. Calcif Tissue Int 105(2):109-124. https://doi.org/ 10.1007/s00223-019-00561-w

46. Siglinsky E, Krueger D, Ward RE, Caserotti P, Strotmeyer ES, Harris TB, Binkley N, Buehring B (2015) Effect of age and sex on jumping mechanography and other measures of muscle mass and function. J Musculoskelet Neuronal Interact 15(4):301-308

47. Whitmer TD, Fry AC, Forsythe CM, Andre MJ, Lane MT, Hudy A, Honnold DE (2015) Accuracy of a vertical jump contact mat for determining jump height and flight time. J Strength Cond Res 29(4):877-881. https://doi.org/10.1519/jsc.0000000000000542

48. Carlos-Vivas J, Martin-Martinez JP, Hernandez-Mocholi MA, Perez-Gomez J (2018) Validation of the iPhone app using the force platform to estimate vertical jump height. J Sports Med Phys Fitness 58(3):227-232. https://doi.org/10.23736/s0022-4707.16. 06664-0

Publisher's note Springer Nature remains neutral with regard to jurisdictional claims in published maps and institutional affiliations. 